\title{
(6) OPEN ACCESS \\ A novel prepless X-ray imaging capsule for colon cancer screening
}

\author{
Nathan Gluck, ${ }^{1,2}$ Beni Shpak, ${ }^{2}$ Rita Brun, ${ }^{3}$ Thomas Rösch, ${ }^{4}$ Nadir Arber, ${ }_{1}^{1,5}$ \\ Menachem Moshkowitz ${ }^{1,5}$
}

- Additional material is published online only. To view this file please visit the journa online (http://dx.doi.org/10. 1136/gutjnl-2015-310893).

'Department of

Gastroenterology, Tel Aviv Medical Centre, affiliated to Sackler Faculty of Medicine, Te Aviv University, Tel Aviv, Israel ${ }^{2}$ Department of

Gastroenterology, Laniado Medical Centre, Netanya, Israel ${ }^{3}$ Department of Gastroenterology, Rambam Health Care Campus, Haifa, Israel

${ }^{4}$ Department of

Interdisciplinary Endoscopy, University Hospital Hamburg Eppendorf, Hamburg, Germany Integrated Cancer Prevention Center, Tel Aviv Medical Centre, affiliated to Sackler Faculty of Medicine, Tel Aviv University, Tel Aviv, Israel

\section{Correspondence to} Professor Nadir Arber, The Integrated Cancer Prevention Center, Tel-Aviv Sourasky Medical Center, 6 Weizmann St., Tel Aviv 6423906, Israel; nadira@tlvmc.gov.il

Received 14 October 2015 Revised 9 November 2015 Accepted 10 November 2015 Published Online First 1 December 2015

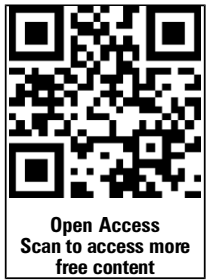

\section{CrossMark}

To cite: Gluck N, Shpak B Brun $\mathrm{R}$, et al. Gut

2016:65:371-373

\section{MESSAGE}

The demand for bowel cleansing hampers participation in screening colonoscopy, while poor preparation impairs adenoma detection. A novel prepless X-ray imaging capsule for colon screening is presented; the capsule emits low dose $\mathrm{X}$-rays, which are scattered by ingested contrast agent mixed with colon contents, and sensed by detectors in the capsule to generate high-resolution threedimensional (3D) imagery of the colon without bowel cleansing. Following preliminary studies with implanted polyps in synthetic colon phantoms, bovine cadaver and live swine, and human experiments with dummy capsules $(n=75), 49$ patients swallowed the capsule with natural excretion in 48 after $73.2 \pm 45.4 \mathrm{~h}$. Pedunculated and sessile polyps were clearly seen and validated by subsequent colonoscopy. Total radiation exposure was ultra low $(0.03 \pm 0.007 \mathrm{mSv})$.

\section{IN MORE DETAIL}

Bowel preparation remains a major obstacle limiting complete uptake of colonoscopy for colon cancer screening. ${ }^{12}$ Therefore any test which does not require preparation would probably increase compliance. ${ }^{3}$ However, the accuracy to depict cancers as well as adenomas should not suffer substantially. A new X-ray capsule is shown in a series of proof-of-concept studies to be safe and effective in $3 \mathrm{D}$ reconstruction of the colonic wall and lumen including identifying adenomatous polyps.

The capsule system includes a short-lived radio isotope within a collimator housing that emits three $\mathrm{X}$-ray beams in all directions, by way of a rotating miniature electric motor as the capsule scans the length of the GI tract. A small volume of ingested radiopaque contrast agent increases the contrast of the colon's walls and differentiates them from stool content. Compton backscattered photons, attenuated by the contrast agent mixed with the colon contents, and X-ray fluorescence photons emitted from the contrast agent, are detected by sensors in the capsule and analysed by on-board electronics and embedded software to estimate the distance from the capsule to the colon wall. These estimations are used to perform 3D image reconstruction of the colon lumen and outer wall (figure 1). In addition, the system generates, as the capsule progresses, an instantaneous track of the capsule position and orientation along the colon, with an accuracy of $\pm 1 \mathrm{~cm}$. This is achieved using additional sensors in the capsule including a 3D accelerometer and a 3D magnetometer, allowing the clinician to localise findings correctly, thus assisting in planning subsequent intervention. The capsule collects these imaging and telemetric data from slices of the colon as it moves and sends them via a radiofrequency link to the capsule positioning system in an external recording unit positioned on the patient's back.

Three types of pilot studies were performed:

Ex vivo studies: The system performance was tested on tissue-equivalent, silicone-based colon phantoms filled with water mixed with $3 \%$ iodinebased contrast. A motorised linear actuator moved the capsule inside the phantom. 'Haustral rings' of the silicone phantom as well polyps $\geq 6 \mathrm{~mm}$ could be visualised. Similar experiments were subsequently performed on a bovine cadaveric colon.

In vivo pig study: The capsule was placed surgically in the terminal ileum of a $70 \mathrm{~kg}$ pig with surgically implanted silicone beads (five measuring $10 \mathrm{~mm}$, five measuring $5 \mathrm{~mm}$ ) in the non-prepped colon to simulate human polyps. After recovery, contrast agent was added during meals. The capsule provided reconstructed images of the porcine colon and had sufficient resolution to detect four/five 10 - $\mathrm{mm}$ polyps as well as $5 \mathrm{~mm}$ polyps (with lower resolution than $10-\mathrm{mm}$ polyps). The two undetected polyps were those most distal (capsule battery had stopped functioning). No adverse effects related to passage of the capsule were noted.

Human studies: (A) To assess safety, 75 dummy capsules were swallowed by volunteers aged $41-70$ years who had no known GI pathology. All capsules were retrieved by the participants at the end of the procedure and were intact when inspected for any leakage or damage.

(B) Scanning capsule study: 49 capsules were swallowed by 46 volunteers aged $45-68$ years (three patients swallowed the capsule on two different occasions). Forty-eight out of the 49 capsules were swallowed and naturally eliminated by the volunteers without any minor or major side effects. In one case, the capsule was retained in the caecum and retrieved during follow-up colonoscopy performed for polypectomy. This participant was asymptomatic. Total patient radiation exposure was $0.03 \pm 0.0007 \mathrm{mSv}$. Image reconstructions allowed 3D views of colonic wall and lumen with the typical contour of different segments (hepatic flexure, triangular shape of the transverse colon). Additionally, no 'haustral rings' were observed during capsule movement. Polyps were detected in several patients and validated later by colonoscopy. Representative examples include a sessile polyp in the ascending colon (figure $2 \mathrm{~A}$ ) and a pedunculated, lobulated polyp in the sigmoid colon (figure 2B). Find more details on online supplementary methods and results. 
Figure 1 Image acquisition by the capsule. Top: Data from Compton backscattering and X-ray fluorescence are combined to estimate the distance from the capsule to the colon walls. Bottom left: Thee-dimensional (3D) reconstruction of the lumen is based on these estimates. Bottom middle: The capsule positioning system presents position and orientation of the capsule to assist in on-line evaluation. Bottom right: Surface and pathway data are fused to create an image reconstruction of the colon.
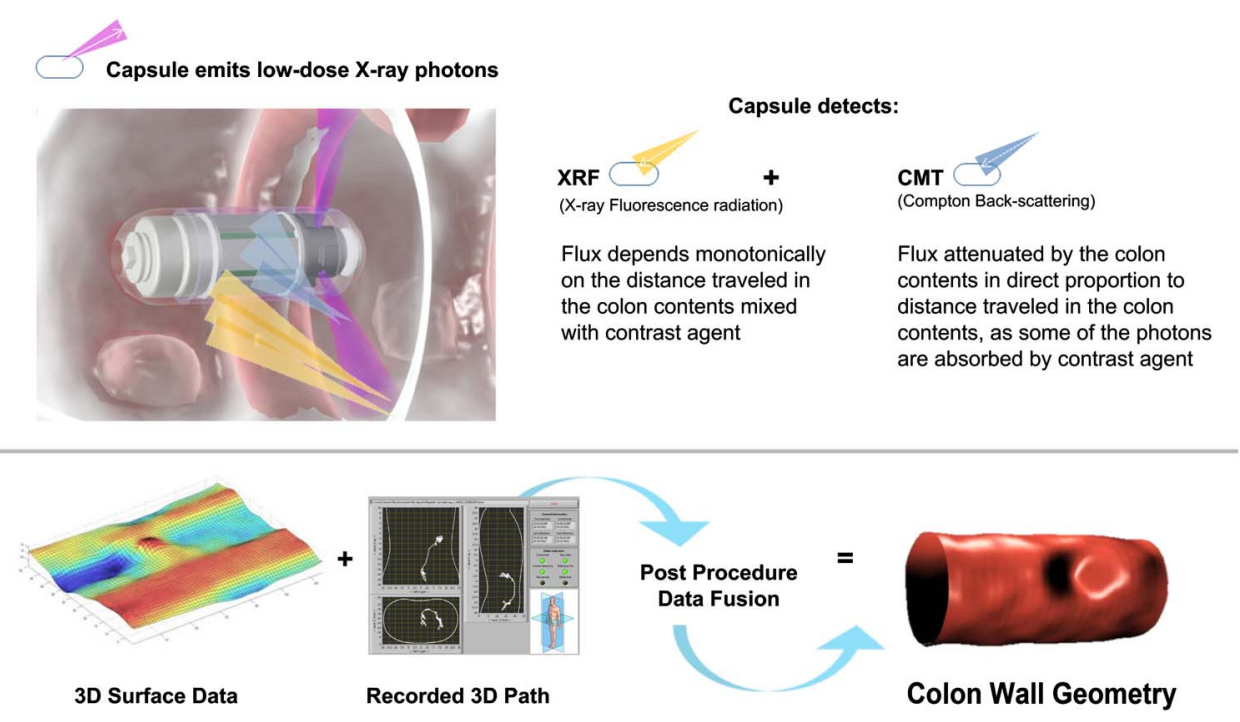

\section{COMMENTS}

A patient-friendly capsule that provides 3D imaging of the colon for colorectal cancer (CRC) screening, without the need for bowel preparation, is described. The capsule uses an X-ray source and correlates Compton backscattering and X-ray fluorescence to estimate circumferential distances from the capsule to the colonic
Figure 2 Reconstruction of polyps. (A) Sessile polyp in the ascending colon. (A) Endoscopic image of a $12 \times 4 \mathrm{~mm}$ sessile polyp (arrow) on a haustrum. (B) Thee-dimensional (3D) trace of the colon by capsule data (anteroposterior view). The blue line marks the segment where a polyp was visualised. (C and D) Reconstruction of the colon segment: tube-type (C) or fillet-type (D). Polyp is marked by arrow. (E) Reconstruction of outer colon surface, polyp visible as indentation (arrow). (B): As in A, depicting a $20 \mathrm{~mm}$ pedunculated, lobulated polyp in the sigmoid colon.
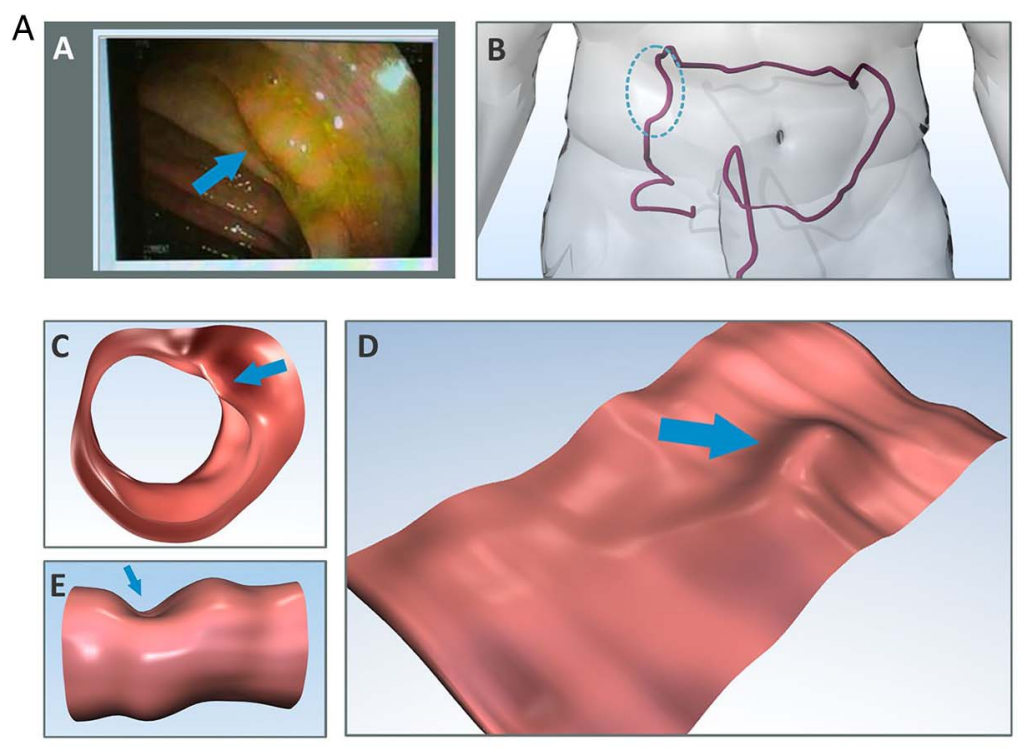

B
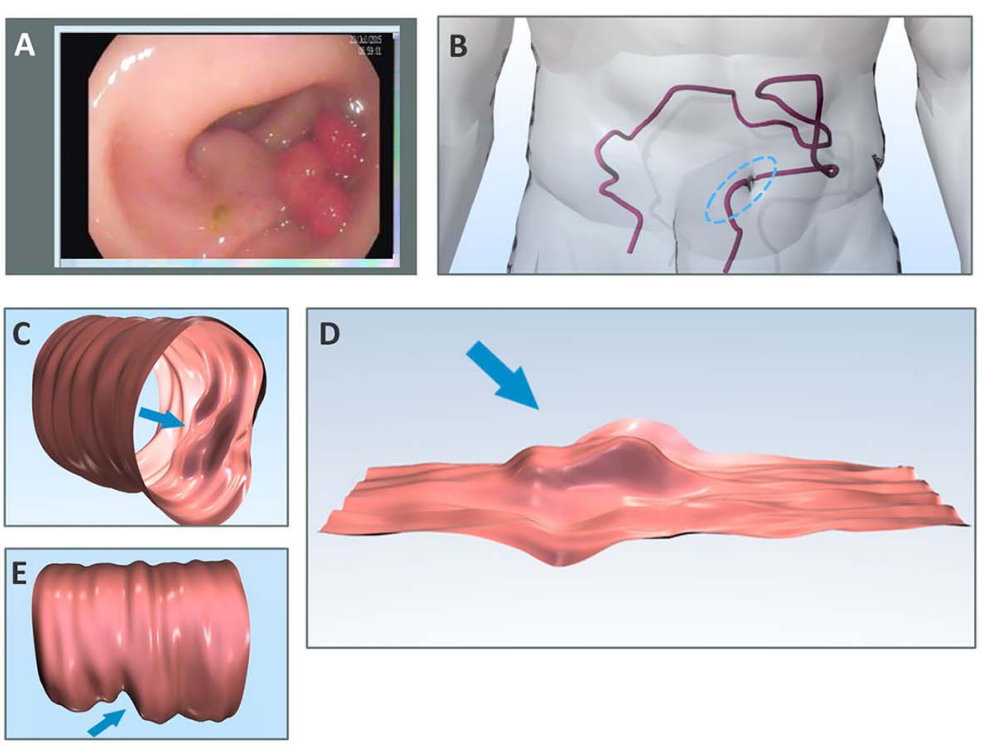
wall. Unlike optical imaging, X-rays that penetrate the colon contents enable the capsule to map the inner topography of the colon surface while 'seeing' through stool in the colon.

The study confirmed the safe passage of the capsule from mouth to rectum in $>120$ subjects with a very low radiation exposure (equivalent to one chest X-ray, 1/200 of CT scan). The study affirmed the ability of the capsule to reconstruct the human colon and to identify polyps without the need for cathartic preparation.

This capsule could hopefully appeal to a large portion of the population that is deterred by bowel preparation and thus increase compliance with structural CRC screening. The low radiation may be advantageous to patients and physicians concerned with higher doses of radiation associated with CT colonography. This capsule will compete with other novel CRC screening methods such as endoscopic colon capsule, stool DNA testing and blood biomarkers. To which extent all these recent methods will detect cancer at an earlier stage and have good performance in detecting precancerous lesions will be seen in further studies.

Reading the imaging data from this capsule and discriminating between false positive and true findings that represent potential lesions in the colon requires a physician with experience in colonoscopy and a solid knowledge of colon anatomy and physiology. There are still many features of image reconstruction and measurement of polyp size and colon diameter which need refinement and, most importantly, need validation compared with colonoscopy. Therefore, it is premature to draw conclusions from this initial uncontrolled trial. Further multicentre studies to validate the performance of this capsule are underway.

Contributors NG, TR and NA contributed to planning, conducting and reporting the findings. BS, RB and TR contributed to conducting the study. MM contributed to planning and conducting the study.

Funding The study was funded by Check Cap, Mount Carmel, Israel Competing interests NA reports receiving consulting fees from Check Cap.

Ethics approval Ethics Committee/Institutional Review Board, Tel-Aviv Sourasky Medical Center and Laniado Hospital.

Provenance and peer review Not commissioned; internally peer reviewed.

Open Access This is an Open Access article distributed in accordance with the Creative Commons Attribution Non Commercial (CC BY-NC 4.0) license, which permits others to distribute, remix, adapt, build upon this work non-commercially, and license their derivative works on different terms, provided the original work is properly cited and the use is non-commercial. See: http://creativecommons.org/ licenses/by-nc/4.0/

\section{REFERENCES}

1 Harewood GC, Wiersema MJ, Melton LJ III. A prospective, controlled assessment of factors influencing acceptance of screening colonoscopy. Am J Gastroenterol 2002;97:3186-94.

2 McLachlan SA, Clements A, Austoker J. Patients' experiences and reported barriers to colonoscopy in the screening context--a systematic review of the literature. Patient Educ Couns 2012:86:137-46.

3 Beebe TJ, Johnson CD, Stoner SM, et al. Assessing attitudes toward laxative preparation in colorectal cancer screening and effects on future testing: potential receptivity to computed tomographic colonography. Mayo Clin Proc 2007;82:666-71. 